\title{
EQUIVALENCE OF THE DEFINING SEQUENCES FOR ULTRADISTRIBUTIONS
}

\author{
SOON-YEONG CHUNG AND DOHAN KIM
}

(Communicated by Palle E. T. Jorgensen)

\begin{abstract}
We prove that $M_{p}^{*} p ! \sim M_{p}$. This result is a stronger version of the conjecture $M_{p}^{*} p ! \supset M_{p}$ which has been given by Komatsu in Ultradistributions I, Structure theorems and a characterization (J. Fac. Sci. Univ. Tokyo Sect. IA 20 (1973), 25-105).
\end{abstract}

Let $M_{p}$ be a sequence of positive numbers with $M_{0}=1$, satisfying the following two conditions :

(M.1) $M_{p}^{2} \leq M_{p-1} M_{p+1}, p=1,2, \ldots$,

(M.3) $\sum_{k=p+1}^{\infty} M_{k-1} / M_{k} \leq H p M_{p} / M_{p+1}, p=1,2, \ldots$, for some $H>0$.

We say that two sequences $M_{p}$ and $N_{p}$ are equivalent and denote it by $M_{p} \sim$ $N_{p}$ if there are constants $A, B>0$, such that

$$
A^{p} N_{p} \leq M_{p} \leq B^{p} N_{p}, \quad p=1,2, \ldots .
$$

The above sequence $M_{p}$ is used to define the various spaces of ultradifferentiable functions and ultradistributions. These spaces are invariant under the equivalence ' $\sim$ ' (see Komatsu [1] for details).

The purpose of this note is to show that $M_{p}^{*} p ! \sim M_{p}$ which refines Theorems 11.5 and 11.8 in Komatsu [1]. Here

$$
M_{p}^{*}=\sup _{t>0} \frac{t^{p}}{\exp M^{*}(t)} \quad \text { and } \quad M^{*}(t)=\sup _{p \in \mathbf{N}} \log \frac{p ! t^{p}}{M_{p}} .
$$

Theorem. Let $M_{p}$ be a sequence as above. Then

$$
M_{p}^{*} \sim \frac{M_{p}}{p !}, \quad p=1,2, \ldots .
$$

Proof. Let $m_{p}=M_{p} / M_{p-1}$ and $l_{p}=p / m_{p}+\sum_{k \geq p}^{\infty} 1 / m_{k}$. Then the sequence $l_{p}$ decreases because $M_{p}$ satisfies (M.1). Since $\bar{M}_{p}$ satisfies (M.3), there is a constant $A \geq 1$ such that

$$
\frac{m_{p}}{p A} \leq \frac{1}{l_{p}} \leq \frac{m_{p}}{p}, \quad p=1,2, \ldots .
$$

Received by the editors July 24, 1991.

1991 Mathematics Subject Classification. Primary 46F05.

Key words and phrases. Defining function, ultradistribution, equivalent.

The first author was partially supported by the GARC-KOSEF, and the second author was partially supported by the Ministry of Education. 
Let $n_{p}=p l_{1} / l_{p}, n_{0}=1$, and $N_{p}=\prod_{j=0}^{p} n_{j}$. Then $N_{p}$ is equivalent to $M_{p}$ and satisfies (M.1), (M.3). Moreover, the sequence $N_{p} / p$ ! also satisfies (M.1). It follows from these facts and Gorny's theorem (Mandelbrojt [2]) that

$$
\frac{N_{p}}{p !}=\sup _{t>0} \frac{t^{p}}{\exp N^{*}(t)} \text {. }
$$

On the other hand, the equivalence $N_{p} \sim M_{p}$ implies that $N_{p} / p ! \sim M_{p} / p$ ! and there are constants $A, B>0$ such that

$$
N^{*}(A t) \leq M^{*}(t) \leq N^{*}(B t), \quad t>0 .
$$

Thus,

$$
\begin{aligned}
\left(\frac{1}{B}\right)^{p} \frac{N_{p}}{p !} & =\sup _{t>0} \frac{t^{p}}{\exp N^{*}(B t)} \leq \sup _{t>0} \frac{t^{p}}{\exp M^{*}(t)}=M_{p}^{*} \\
& \leq \sup _{t>0} \frac{t^{p}}{\exp N^{*}(A t)}=\left(\frac{1}{A}\right)^{p} \frac{N_{p}}{p !}, \quad p \in \mathbf{N} .
\end{aligned}
$$

Therefore, it follows that

which completes the proof.

$$
M_{p}^{*} \sim \frac{N_{p}}{p !} \sim \frac{M_{p}}{p !}
$$

\section{REFERENCES}

1. H. Komatsu, Ultradistributions I, Structure theorems and a characterization, J. Fac. Sci. Univ. Tokyo Sect. IA 20 (1973), 25-105.

2. S. Mandelbrojt, Séries adhérentes régularisation des suites, applications, Gauthier-Villars, Paris, 1952.

Department of Mathematics, Duksung Women's University, Seoul 132-714, Korea

Department of Mathematics, Seoul National University, Seoul 151-742, Korea 